\section{Automatic extraction of the mid-facial plane for cranio- maxillofacial surgery planning}

\author{
E. De Momi, J. Chapuis, I. Pappas, G. Ferrigno, W. Hallermann, A. Schramm, M. \\ Caversaccio: Automatic extraction of the \\ mid-facial plane for cranio-maxillofacial surgery planning. Int. J. Oral Maxillofac. \\ Surg. 2006; 35: 636-642. (C) 2006 International Association of Oral and Maxillofacial \\ Surgeons. Published by Elsevier Ltd. All rights reserved.
}

Abstract. Recently developed computer applications provide tools for planning cranio-maxillofacial interventions based on 3-dimensional (3D) virtual models of the patient's skull obtained from computed-tomography (CT) scans. Precise knowledge of the location of the mid-facial plane is important for the assessment of deformities and for planning reconstructive procedures. In this work, a new method is presented to automatically compute the mid-facial plane on the basis of a surface model of the facial skeleton obtained from CT. The method matches homologous surface areas selected by the user on the left and right facial side using an iterative closest point optimization. The symmetry plane which best approximates this matching transformation is then computed. This new automatic method was evaluated in an experimental study. The study included experienced and inexperienced clinicians defining the symmetry plane by a selection of landmarks. This manual definition was systematically compared with the definition resulting from the new automatic method: Quality of the symmetry planes was evaluated by their ability to match homologous areas of the face. Results show that the new automatic method is reliable and leads to significantly higher accuracy than the manual method when performed by inexperienced clinicians. In addition, the method performs equally well in difficult trauma situations, where key landmarks are unreliable or absent.

\author{
E. De Momi ${ }^{1,2}$, J. Chapuis ${ }^{1}$, \\ I. Pappas ${ }^{1}$, G. Ferrigno ${ }^{2}$, \\ W. Hallermann ${ }^{3}$, A. Schramm ${ }^{4}$, \\ M. Caversaccio ${ }^{1,5}$ \\ ${ }^{1}$ M.E. Müller Institute for Surgical Technology \\ and Biomechanics, University of Bern, \\ Switzerland; ${ }^{2}$ TBM Lab - Bioengineering \\ Department, Politecnico di Milano, Italy; \\ ${ }^{3}$ CMF-Department, Inselspital, University of \\ Bern, Switzerland; ${ }^{4}$ Department of Oral and \\ Maxillofacial Surgery, University Clinic \\ Freiburg, Germany; ${ }^{5}$ ENT-Department, \\ Inselspital, University of Bern, Switzerland
}

Key words: mid-facial; mid-sagittal; plane; symmetry; skull; cranio-maxillofacial; surgery; computer aided.

Accepted for publication 27 January 2006 Available online 20 March 2006
Corrective and reconstructive cranio-maxillofacial (CMF) surgery aims to correct facial deformities, restore function and reestablish facial symmetry and harmony in pathologies that may be caused by congenital malformations, tumour-related or trauma-related deformities. Recently developed computer applications provide tools for planning CMF interventions based on 3-dimensional (3D), virtual models of the patient's skull obtained from preoperative computed-tomography (CT) scans ${ }^{3,6,7,13}$. Interactive tools for graphical manipulation of the virtual skull models enable the surgeon to perform 3D cephalometric measurements, simulate osteotomies, relocate or mirror bone segments ${ }^{4,5,16}$, insert implants and grafts, and simulate 3D soft-tissue deformations $^{17,18}$. Precise knowledge of the location of the mid-facial plane (also referred to as mid-sagittal plane, or symmetry plane) is essential for the assessment of facial deformities and for the planning of reconstructive procedures ${ }^{12}$. The midfacial plane allows correction of the head tilt in the image data and facilitates visual 
and numerical assessment of symmetry. In addition, the mid-facial plane can be used in asymmetrical deformities to mirror the healthy facial side: The result can then be employed as a template for correction of the affected side. The problem of computing the mid-facial plane is also fundamental in other areas of medicine, for example for the study of functional and anatomical brain symmetry ${ }^{9,10}$.

Several methods have been proposed to compute the mid-facial plane using volumetric image datasets. A straightforward way of defining the plane is to select a number of anatomical landmarks in the dataset, which either lie directly on the plane or are on equal distances on either side of it. Manual selection of landmarks, however, is time-consuming, as it requires great care and attention during the selection process. In addition, the result depends on availability and visibility of the anatomical landmarks and on the ability of the user to identify them. A second category of methods is based on maximizing a measurement which quantifies facial symmetry. This class of methods proceeds by optimizing the measurement for the entire image volume. A commonly used measurement is cross-correlation of intensity values of either sides of the face, in the coronal and axial 2D slice images ${ }^{9,10}$ or directly in the 3D images ${ }^{1,15}$. Other measurements of symmetry using image intensities have been reported; in particular, methods based on edge extraction ${ }^{10}$ and extended Gaussian image transformation ${ }^{14}$.

The goal of this work was to develop and validate a new method for computing the mid-facial plane, which operates with surface models of the facial skeleton. Surface models offer the advantage that only the segmented anatomical structures, the facial skeleton's surface in this particular case, are taken into account in the computation. In addition, the area to be considered can be narrowed to a selected area of the anatomy made by the user. To compute the symmetry plane the method automatically matches 2 user-selected corresponding surface patches on either side of the face. The symmetry plane can thus be optimized for specific regions on the facial skeleton, which are selected by the user. Definition of the plane can therefore be adapted to each individual case, in a much finer way than methods based on the full image volume. In addition, the surface-based method allows the user to disregard areas that are asymmetric due to trauma or other pathologies and ignore internal structures of the skull, which are irrelevant to external, visible facial symmetry.
In this paper, results are presented of an experimental study comparing the new surface-based symmetry plane definition with the landmark-based definition.

\section{Methods}

The first step in either the surface-based or the landmark-based method is the generation of a surface model suitable for subsequent visualization and processing. Any standard CT dataset can be used as input. On the basis of Hounsfield standard values, a threshold value is chosen to segment the bone. This threshold value is used to generate an iso-surface using the marching cubes algorithm ${ }^{11}$. The result is a detailed surface model of the skeleton, typically composed of several million polygons. To allow handling such a model at interactive rates, a locally adaptive decimation algorithm is used in order to reduce the number of polygons to approximately 150,000-250,000 per skull model ${ }^{8}$. For the experimental study, the CT datasets were obtained from a helical scanner with $1.25 \mathrm{~mm}$ slice thickness (settings: $140 \mathrm{kV}$ and $120 \mathrm{~mA}$; model: LightSpeed Ultra, GE Medical Systems, Milwaukee, USA).

The surface-based method for automatic mid-facial plane computation was developed on a platform for CMF planning and navigation. The CMF platform currently includes tools for 3D cephalometry, mirroring, virtual osteotomies, bone segment relocation and manipulation, graft insertion, and mid-facial plane computation (see Fig. 1). The platform even supports intraoperative guidance for the execution of the virtual surgical plans thanks to a specific surgical navigation module.

\section{Landmark-based method}

In the landmark-based method, the user selects a minimum of 3 anatomical landmarks on the bone surface model. If 3 landmarks are chosen, they uniquely define the plane. If more than 3 landmarks are chosen, the plane is computed using a least-squares algorithm, which minimizes the squared landmark-plane distances. Landmarks either lie on midline structures or consist in bilateral pairs, as shown in Fig. 2, in which case the algorithm takes the mean of the 2 points into account.

\section{Surface-based method}

In the surface-based method, the user is asked to select a pair of homologous surface areas on the left and right facial sides on the bone surface model of the skull by 'painting' these areas using the computer mouse, as shown in Fig. 3. The algorithm then automatically computes the best symmetry plane for the selected areas. Computation of the plane is done by matching 1 of the 2 selected surface patches to the other using an iterative closest point optimization algorithm ${ }^{2}$ (ICP). To do so, one of the patches is mirrored on a symmetry plane that is obtained by an initial guess. The initial guess is made by taking the 'centres of gravity' of each surface patch: The mean of the 2 'centres of gravity' is the origin of the plane, and connecting a line segment between them gives the plane normal. ICP iteratively finds the rigid transformation which minimizes the Euclidean distances between 2 sets of points. The mid-facial plane is the mirroring plane, which best approximates the summed transformations of initial mirroring (based on initial guess) followed by ICP matching. The plane is computed in a similar way as for the initial guess, but this time the centre of gravity of the same surface patch is used: Mirrored and matched points are connected to the intact points, giving the final plane origin and normal vector (see Table 1).

\section{Experimental protocol}

Quantitative comparison of landmarkbased and surface-based methods for the computation of the mid-facial symmetry plane was carried out as follows: First, as a control experiment, the surface-based method was evaluated on 6 artificial, perfectly symmetric skeletal models. These artificial models were constructed by virtually splitting a plastic model in 2 and replacing 1 side by the mirror of the other side.

Subsequently the 2 methods were compared on 12 skull models obtained from CT datasets of patients devoid of any particular CMF deformities (CT datasets were provided by the Inselspital, University of Bern, Switzerland). Two experienced and 2 inexperienced CMF surgeons were asked to participate in the experimental study. The study started with landmark selection: The 4 surgeons were asked to choose the landmarks they considered relevant for the definition of the mid-facial plane, in each of the 12 cases. This implied manual selection of a total of 48 planes. An average number of 5 landmarks were selected per plane. For each case, after selection of landmarks, the resulting plane was shown graphically in the $3 \mathrm{D}$ view of the model. Surgeons were allowed 1 correction of their landmark selections if they considered it necessary after viewing the initial results. 
(a)
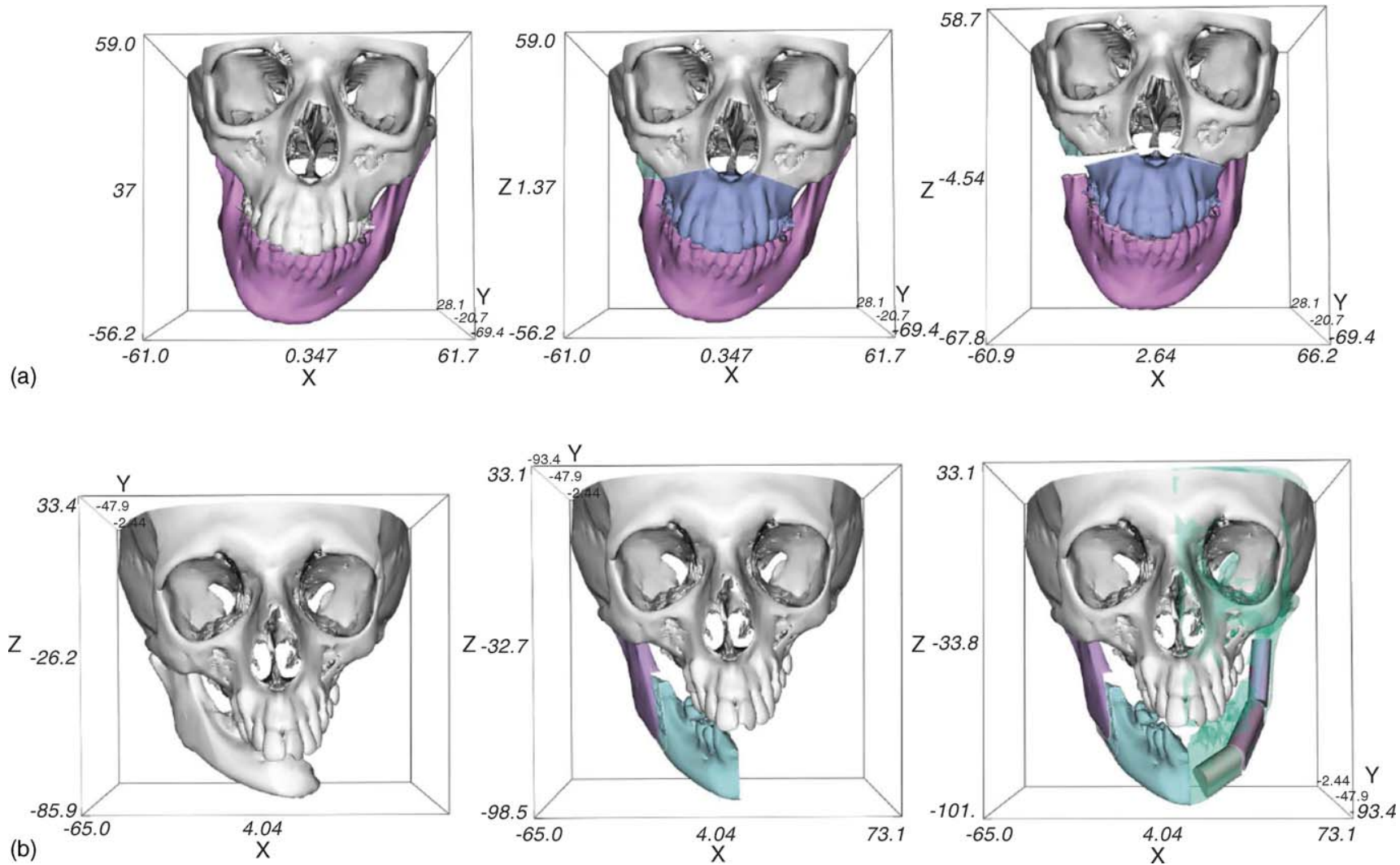

Fig. 1. Virtual planning of CMF interventions. (a) Steps in virtual osteotomy planning for a bi-maxillary corrective procedure in the case of a unilateral malformation and (b) steps in virtual planning of mandibular sagittal split osteotomy and complete hemi-mandibular reconstruction using simulated tibial grafts. The mirrored contralateral side has been used as template for designing the graft; note that further intervention would be needed to fully correct the asymmetry.

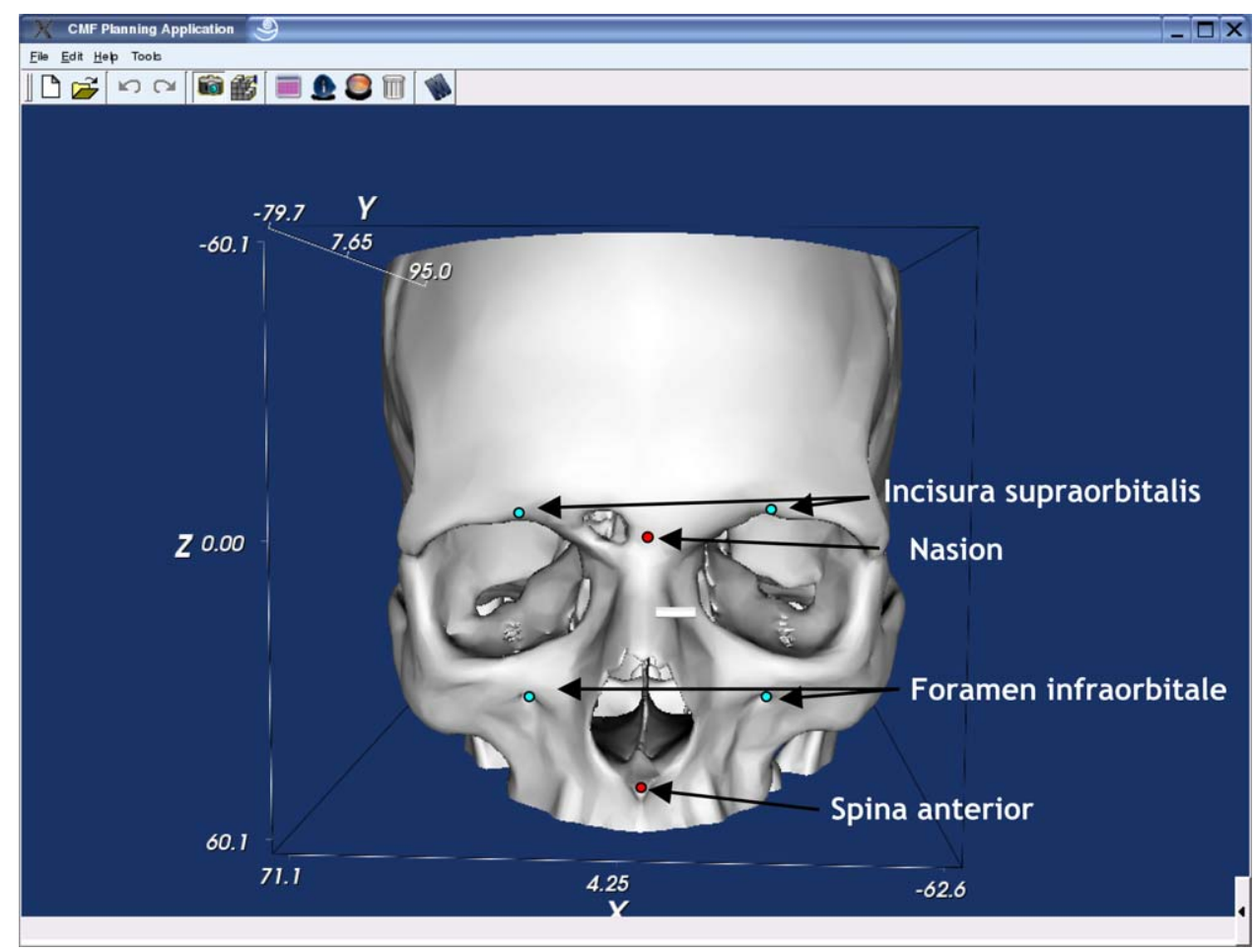

Fig. 2. Landmark-based method for symmetry plane computation: examples of possible landmark choices, midline structures (red points) and bilateral symmetrical points (blue points) on a coronal view of the model. 


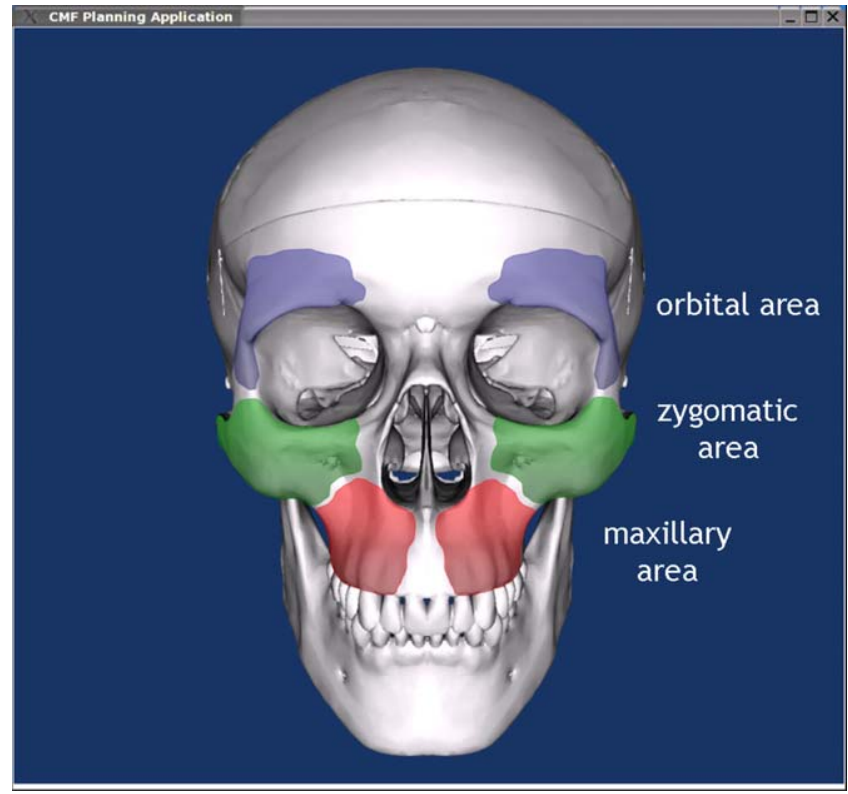

Fig. 3. Surface-based method for symmetry plane computation. The 3 facial areas defined for the systematic comparative study: orbital, zygomatic and maxillary.

In a second step for comparison, surface regions were 'painted' for automatic computation of the mid-facial plane using the surface-based method. To allow systematic comparison of the results across all datasets, identical anatomical areas were selected: orbital, zygomatic and maxillary, as shown in Fig. 3.

For both methods, the quality of the computed symmetry plane was evaluated by the ability of the plane to mirror homologous facial areas on to each other. The symmetry measurement employed was the following: orbital, zygomatic and maxillary areas were mirrored using the computed symmetry plane and quality of the match was evaluated by the mean Euclidean distance between the mirrored surface patch and its untouched counterpart, as given by the Eq. (1):

where $x_{\mathrm{p}}, y_{\mathrm{p}}$ and $z_{\mathrm{p}}$ are the coordinates of the model vertices from the mirrored surface patch, $x_{\mathrm{t}}, y_{\mathrm{t}}$ and $z_{\mathrm{t}}$ are the coordinates of the closest points on untouched counterpart and $N_{\mathrm{p}}$ the total number of vertices.

Time performance of both methods was also evaluated: For the landmark-based method, the time spent by surgeons in selecting the landmarks as well as plane computation times were considered. For the surface-based method, times required for bilateral selection of surface patches and for computation of the symmetry plane were considered.

A statistical analysis of the results was carried out, using the non-parametric Fisher LSD test $(P<0.05)$. This analysis gave us a basis to compare the 2 methods for obtaining the mid-facial plane (landmark-based and surface-based).

\section{Results}

After computation of a particular midfacial plane, a graphical representation of that plane was shown in the $3 \mathrm{D}$ view together with the mirroring of the facial skeleton using the plane (Fig. 4). Quantitative results of control and comparative experiments are given below.

\section{Control study}

As explained above, in the control experiment, the surface-based method was tested on 6 artificially constructed, perfectly symmetric skulls. Quality of the computed symmetry plane was evaluated by its ability to mirror homologous areas on to each other, as expressed by Eq. (1).

The results are summarized in Fig. 5. Along the horizontal axis, results are grouped according to the selected area: (1) orbital; (2) zygomatic; (3) maxillary and (4) merged orbital, zygomatic and maxillary areas. Data columns indicate the measurement of error, i.e. the median value and quartile deviations of distances between areas mirrored with the computed plane and their counterparts, for each of the defined anatomical regions. These results indicate that the surface-based method performs as expected. Measured errors (average value below $0.02 \mathrm{~mm}$ ) are negligible.

\section{Experimental study}

In the manual plane selection experiment, users selected 4-6 anatomical landmarks to define the plane. Most frequently chosen anatomical landmarks were the following: Nasion, spina nasalis anterior, spina nasalis posterior, vomer, crista galli, sella turcica. Users corrected their landmark selections after viewing the result in approximately $20 \%$ of the cases. The time required to obtain a satisfactory result was on average $10 \mathrm{~min}$ for each skull, including computation of the symmetry plane (which was quasi instantaneous: less than

Table 1. Description of the steps in the surface-based computation method

\begin{tabular}{ll}
\hline Step number & The user selects with the mouse cursor used as a graphical painting tool 2 homologous areas on the left and right \\
\hline 1 & side of the skull, called the Point Set (PS) and Model Set (MS). \\
& The PS is mirrored by reflection symmetry $\left(\mathrm{M}_{1}\right)$ on a default symmetry plane which is placed initially (default) \\
& half way between the 2 areas. The mirrored PS (given by PSm $=\mathrm{M}_{1} \cdot \mathrm{PS}$ ) is then registered to the MS using an \\
& iterative ICP algorithm ${ }^{16}$ implementation. The obtained registration matrix (R) is then applied to the mirrored PS \\
& (PSm) yielding the registered mirrored PS (PSmreg), given by: PSmreg $=\mathrm{R} \cdot \mathrm{M}_{1} \cdot \mathrm{PS}$. \\
& After application of the mirroring and registration transformations to the PS, the mid-facial plane is computed \\
& from the PS and PSmreg as the plane normal to the line segment connecting gravity centres of PS and PSmreg and \\
& passing through the midpoint of this line segment.
\end{tabular}

Note: The algorithm performs poorly if the selected surface areas are exceedingly small or flat, since ICP surface matching must be constrained in all 6 degrees of freedom (translations and rotations). A warning is issued to the user if the selected surface areas are not suitable. 


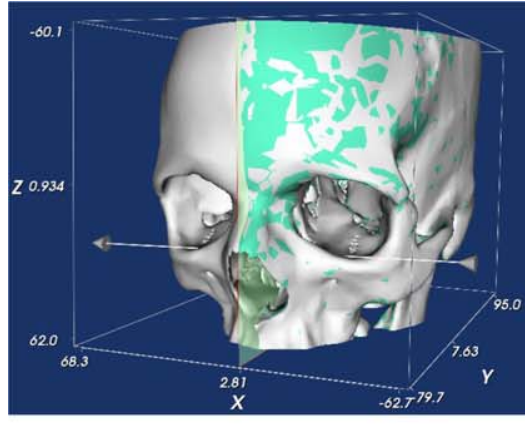

(a)

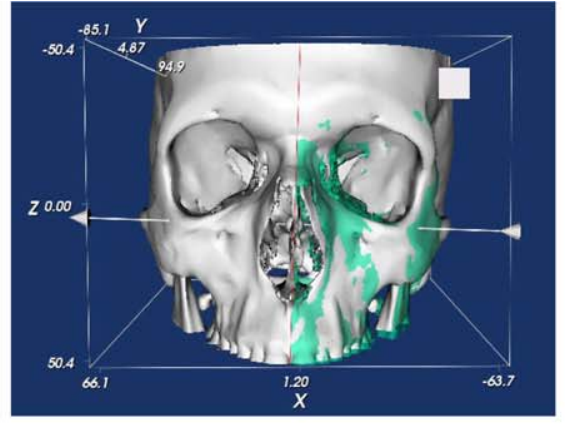

(b)

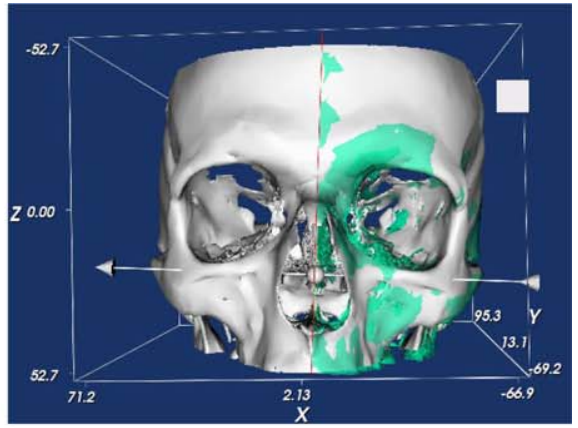

(c)

Fig. 4. Illustration of results obtained with the surface-based method. Examples of results obtained with surface-based computation of the midfacial symmetry plane for: (a) a perfectly symmetric skull, and (b) and (c) 2 patient skulls. The computed symmetry plane is used to mirror half of the facial skeleton on to the opposite side: the green overlays show the mirrored surface in locations where it lies above the real surface. The arrow indicates the symmetry plane normal vector.

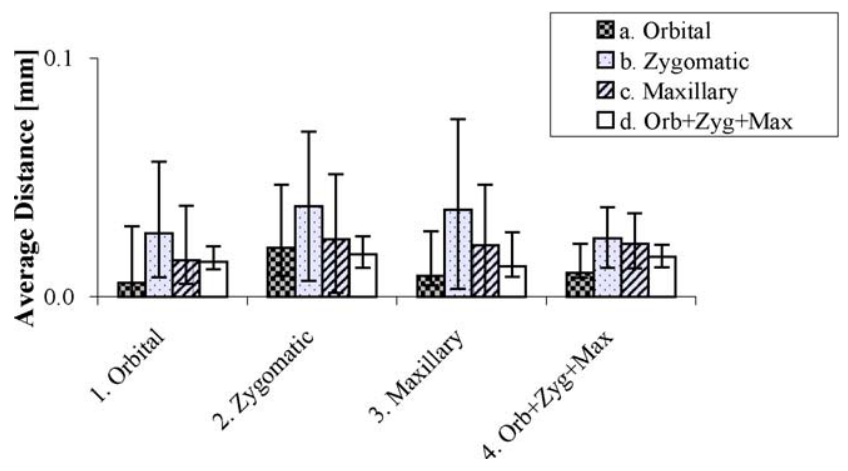

Fig. 5. Results on 6 perfectly symmetric skulls: experimental results of the surface-based method on 6 artificial, perfectly symmetric skulls. On the horizontal axis, results are grouped according to selected surface area: (1) orbital, (2) zygomatic, (3) maxillary and (4) merged orbital, zygomatic and maxillary areas. Data columns indicate the measurement of error, i.e. the median value and quartile deviations of distances between areas mirrored with the computed plane and their counterparts, for each of the defined anatomical regions.

$1 \mathrm{~s}$ on a standard PC). In the surface-based plane computation experiment, the time required for selection of bilateral skull areas and for computation of the symmetry plane was approximately 2 min.

Quantitative results for both methods are summarized in Fig. 6, results on the horizontal axis are grouped according to the selected area. Here again, data columns indicate, for each resulting symmetry plane, the median value and quartile deviations of the distance error measurement, computed for all 4 defined anatomical areas.

Statistical testing yielded the following significant differences (see Table 2 for numerical values):

(i) Surface-based definition using orbital or zygomatic areas leads to a more accurate symmetry plane in the orbital area than landmark-based definition, whether performed by experienced or inexperienced surgeons (Fig. 6: compare $1 \mathrm{a}$ with $5 \mathrm{a}$ and $6 \mathrm{a}$ and compare $2 b$ with $5 b$ and $6 b$ ). (ii) Surface-based definition using maxillary areas or merged orbital, zygomatic and maxillary areas leads to a more accurate symmetry plane in the defined anatomical regions. orbital area than landmark-based definition, when performed by inexperienced surgeons, while there is no significant statistical difference when performed by experienced surgeons (Fig. 6: compare $3 \mathrm{c}$ with $5 \mathrm{c}$ and $6 \mathrm{c}$ and compare $4 \mathrm{~d}$ with $5 \mathrm{~d}$ and $6 \mathrm{~d}$ ).

\section{Discussion}

In the control study measured errors are negligible. It is thus concluded that the automatic method computes the symmetry plane correctly in the ideal case of symmetric skulls.

In the experimental study the manual landmark-based method was compared with the automatic surface-based method for the extraction of the mid-facial plane on 12 CT datasets of patients, who did not suffer from pathologic skull deformities. Results obtained from the experimental

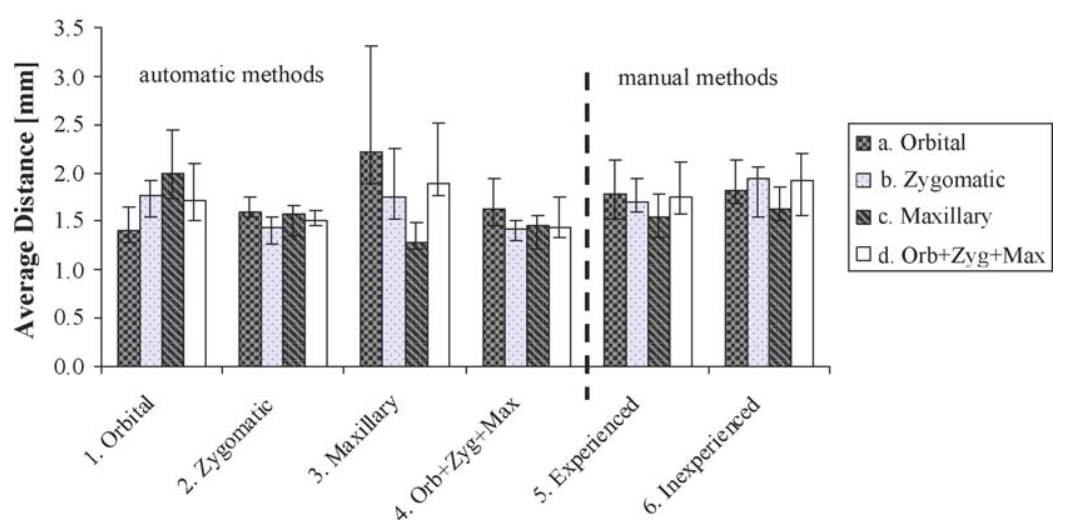

Fig. 6. Results on 12 patient skulls: experimental results on the 12 patient skulls. On the horizontal axis, for surface-based definitions, results are grouped according to selected areas: (1) orbital, (2) zygomatic, (3) maxillary, and (4) merged orbital, zygomatic and maxillary areas; for manual definitions, results are divided into (5) experienced and (6) inexperienced groups. Data columns indicate the measurement of error, i.e. the median value and quartile deviations of distances between areas mirrored with the computed plane and their counterparts, for each of the 
Table 2. Statistical analysis of the experimental results obtained with the 12 processed patient datasets

\begin{tabular}{|c|c|c|c|}
\hline & $\begin{array}{l}\text { Automatic } \\
\text { method }\end{array}$ & $\begin{array}{l}\text { Expert } \\
\text { selection }\end{array}$ & $\begin{array}{l}\text { Non-expert } \\
\text { selection }\end{array}$ \\
\hline \multicolumn{4}{|c|}{$\begin{array}{l}\text { Non-parametric Fischer LSD test }(P<0.05) \\
\text { (a) Orbital area }\end{array}$} \\
\hline $\begin{array}{l}\text { Automatic method } \\
\text { Expert selection } \\
\text { Non-expert selection }\end{array}$ & $\begin{array}{l}\text { - } 0.008 \\
\bullet \mathbf{0 . 0 2 6}\end{array}$ & $\begin{array}{l}\bullet \mathbf{0 . 0 0 8} \\
/ \\
0.675\end{array}$ & $\begin{array}{l}\bullet \mathbf{0 . 0 2 6} \\
0.675 \\
/\end{array}$ \\
\hline $\begin{array}{l}\text { (b) Zygomatic area } \\
\text { Automatic method } \\
\text { Expert selection } \\
\text { Non-expert Selection }\end{array}$ & $\begin{array}{l}\text { - } 0.018 \\
\bullet 0.004\end{array}$ & $\begin{array}{l}\bullet \mathbf{0 . 0 1 8} \\
/ \\
0.622\end{array}$ & $\begin{array}{l}\bullet \mathbf{0 . 0 0 4} \\
0.622 \\
/\end{array}$ \\
\hline $\begin{array}{l}\text { (c) Maxillary area } \\
\text { Automatic method } \\
\text { Expert selection } \\
\text { Non-expert Selection }\end{array}$ & $\begin{array}{l}/ \\
0.070 \\
\bullet \mathbf{0 . 0 0 5}\end{array}$ & $\begin{array}{l}0.070 \\
/ \\
0.299\end{array}$ & $\begin{array}{l}\mathbf{0} \mathbf{0 . 0 0 5} \\
0.299 \\
/\end{array}$ \\
\hline $\begin{array}{l}\text { (d) Orb }+ \text { Zyg }+ \text { Max Are } \\
\text { Automatic method } \\
\text { Expert selection } \\
\text { Non-expert Selection }\end{array}$ & $\begin{array}{l}/ \\
0.054 \\
\bullet \mathbf{0 . 0 2 6}\end{array}$ & $\begin{array}{l}0.054 \\
/ \\
0.753\end{array}$ & $\begin{array}{l}\bullet \mathbf{0 . 0 2 6} \\
0.753 \\
/\end{array}$ \\
\hline
\end{tabular}

For each of the areas used in the automatic method (a, b, c and d) the hypothesis stated that the automatic method leads to a different result to the manual methods. The table gives the probability values of that hypothesis being wrong. Values highlighted in bold indicate pairs of results that were found to be significantly different $(P<0.05)$.

study showed that both methods were sufficiently reliable and fast. Mid-facial planes computed with each method were of comparable quality. Statistical analysis of the results indicated that if the aim is to find the optimal symmetry plane for a certain anatomical area (e.g. the zygomatic area), best results are obtained with the surface-based method applied on that precise area. In fact, the human face being imperfectly symmetric, there is no single and unique symmetry plane which can describe facial symmetry. Depending on which facial features are considered, the plane of symmetry is different. Unlike algorithms that compute the mid-sagittal plane using the full $\mathrm{CT}$ or MR image dataset, the presented surface-based method allows symmetry plane computation for a specific anatomic region, according to each individual case and need. Besides, methods using the full CT or MR can be biased due to possible asymmetries in the image volume that are not related to face symmetry. Similarly, in serious pathological cases, where key landmarks for the definition of the symmetry plane are unreliable or even absent, the surface-based method offers a valuable alternative: Computation of the plane can be based on intact regions.

Finally, the method is neither restricted to skeletal models, nor to the face: It can be applied to any surface model and in any anatomical area of the body. It could be used, for example, with a model of the facial skin obtained with an optical scanner, or in the pelvic area, to assist during reconstruction planning with accurate mirrored templates. Another potential application of the method is in education: The study showed that the automatic surfacebased method performed on average better than the inexperienced surgeons and delivered results that were very close to the experienced surgeons. Thus, the method could also serve as an independent expert reference.

Acknowledgements. This work was supported by the Swiss National Science Foundation (http://www.co-me.ch) and by the grant of the Interpolytechnic $\mathrm{PhD}$ School of Biomedical Engineering and Biomechanics (Torino-Milano-Bari, Italy).

\section{References}

1. Ardenaki BA, Kershaw J, Braun M, KanNo I. Automatic detection of the mid-sagittal plane in 3-D brain images. IEEE Trans Med Imaging 1997: 16: $947-$ 952.

2. BESL PJ, McKay ND. A method for registration of 3-D shapes. IEEE Trans Pattern Anal Machine Intell 1992: 14: 239-256.

3. Caversaccio M, Bächler R, Lädrach $\mathrm{K}$, Schroth G, Nolte LP, HäUsler R. The "Bernese" frameless optical computer aided surgery system. Comput Aided Surg 1999: 4: 328-334.
4. Chapuis J, Rudolph T, Borgesson B, De Momi E, Pappas IP, Hallermann W, Schramm A, Caversaccio M. 3D surgical planning and navigation for $\mathrm{CMF}$ surgery. Proc SPIE Medical Imaging 2004: 5367: 403-410.

5. Chapuis J, Rudolph T, Pappas I, CaverSACCIO M. Transferring the 3D Preoperative Plan to Intraoperative Navigation in CMF Surgery. 2nd Annual Meeting of the German Society for Computer and Robotic Assisted Surgery, CURAC, Nürnberg, Germany, November, 2003.

6. Gellrich N, Schramm A, Hammer B, Rojas S, Cufi D, Lagreze W, SchmelZEISEN R. Computer assisted secondary reconstruction of unilateral posttraumatic orbital deformity. Plast Reconstr Surg 2002: 110: 1417-1429.

7. Hassfeld S, Muhling J. Computer assisted oral and maxillofacial surgery - a review and an assessment of technology. Int J Oral Max Surg 2001: 30: 2 13.

8. Heckbert P, Garland M. Optimal triangulation and quadric-based surface simplification. J Comp Geom Theory Appl 1999: 14: 49-65.

9. Junck L, Moen JG, Hutchins GD, Brown MB, KUHL DE. Correlation methods for the centering, rotation and alignment of functional brain images. $\mathrm{J}$ Nuclear Med 1990: 3: 1220-1226.

10. Liu Y, Collins RT, Rothfus WE. Robust midsagittal plane extraction from normal and pathological 3-D neuroradiology images. IEEE Trans Med Imaging 2001: 20: 175-192.

11. Lorensen WE, Cline HE. Marching cubes: a high-resolution 3D surface construction algorithm. J Nucl Med 1987: 21 : 163-169.

12. O'Grady K, Antonyshyn O. Facial asymmetry: three-dimensional analysis using laser surface scanning. Plast Reconstr Surg 1999: 104: 928-937.

13. Schramm A, Gellrich N, Gutwald R, Schipper J, Bloss $\mathrm{H}$, Hustedt $\mathrm{H}$, SCHMELZEISEN R. Indications for computer-assisted treatment of cranio-maxillofacial tumours. Comp Aided Surg 2000: 5: 343-352.

14. Sun C, Sherrah J. 3D symmetry detection using the extended Guassian image. IEEE Trans Pattern Anal Machine Intell 1997: 19: 164-168.

15. Thirion JP, Prima S, Subsol G, ROBERTS N. Statistical analysis of normal and abnormal dissymmetry in volumetric medical images. Med Image Anal 2000: 4: 111-121.

16. Troulis MJ, Everett P, Seldin EB, Kikinis R, Kaban LB. Development of a three-dimensional treatment planning system based on computer tomographic data. Int J Oral Maxillofac Surg 2002: 31: 349-357.

17. Xia J, Samman N, Yeung RW, Shen SG, Wang D, IP HH, Tideman H. Three- 
dimensional virtual reality surgical Address: planning and simulation workbench for orthognathic surgery. Int $\mathrm{J}$ Adult Orthod Orthognath Surg 2000: 15: 265282.

18. Zachow S, Gladilin E, Sader R, ZeilhoFER HF. Draw \& cut: Intuitive 3D osteotomy planning on polygonal bone models. In: Proceedings of the Computer Assisted Radiology and Surgery. London, CARS 2003: 362-369.
Address:

Marco Caversaccio

ME Müller Institute for

Surgical Technology and Biomechanics and Department of ORL

Head and Neck Surgery

University of Bern
Freiburgstrasse

CH-3010 Bern

Switzerland

Tel: +41316324174

Fax: +4131632 4900 .

E-mail: marco.caversaccio@insel.ch 